\title{
Investigation of the transverse beam dynamics in the thermal wave model with a functional method
}

\author{
Ji-ho Jang 1 , Yong-sub Cho, Hyeok-jung Kwon \\ Korea Atomic Energy Research Institute, Daejeon 305-353, Korea
}

\begin{abstract}
We investigated the transverse beam dynamics in a thermal wave model by using a functional method. It can describe the beam optical elements separately with a kernel for a component. The method can be applied to general quadrupole magnets beyond a thin lens approximation as well as drift spaces. We found that the model can successfully describe the PARMILA simulation result through an FODO lattice structure for the Gaussian input beam without space charge effects.
\end{abstract}

PACS number(s): 29.27.-a, 29.27.Eg

Key Words: Transverse Beam Dynamics, Thermal Wave Model, Functional Method

\footnotetext{
${ }^{1}$ jangjh@kaeri.re.kr
} 
The thermal wave model is an efficient way to study the beam dynamics of relativistic charged particles. The Schrödinger-type equation in the model governs the time evolution of the beam wave function whose squared magnitude is proportional to the particle number densities[1. The model has successfully explained the filamentation of a particle beam and the self-pinching equilibrium in collisionless plasma[2]. It was also used to estimate the luminosity in a linear collider where a spherical aberration was present [3]. The model can also provide some insight into a halo formation by introducing a Gaussian slit 4 .

Transverse beam dynamics in a one spatial dimension is another application area of the thermal wave model. In Ref. [5], the authors investigated the beam wave function through a quadrupole magnet with sextupole and octupole perturbations followed by a long drift space under a thin lens approximation. There is also a paper on the phase space behavior of particle beams in the transverse directions where the Wigner and Husimi functions are used as the phase space distribution functions [6].

In this work, we investigate the transverse beam dynamics in a two dimensional trace $\left(x-x^{\prime}\right.$ or $\left.y-y^{\prime}\right)$ space in the thermal wave model by using the functional integral method [7. Because the method can be extended to general lattice structures including quadrupole magnets beyond a thin lens approximation limit and it can treat the beam optical elements individually, it is possible to systematically analyze a beam motion in a realistic environment such as an FODO lattice. We found that the model can successfully explain the PARMILA 8] simulation results with Gaussian input beams in a two dimensional trace space under the condition that the space charge effects are negligible. We note that this method can explain a low energy particle behavior as well as the relativistic motion of the charged particles if the important interactions are related to the external linear optical elements such as the quadrupole magnets and the random motion described by a beam emittance.

In the thermal wave model, the time evolution of the beam wave function for the relativistic charged particles can be described by the Schrödinger-type equation in the transverse directions. Because the beam dynamics are usually described in the two dimensional trace $\left(x-x^{\prime}\right.$ or $\left.y-y^{\prime}\right)$ space, it is important to see whether the one dimensional version of the equation can explain the beam dynamics in the projected space or not. The one dimensional Schrödinger-type equation in $x$ direction is given by

$$
i \epsilon_{x} \frac{\partial \psi_{x}(x, z)}{\partial z}=-\frac{\epsilon_{x}^{2}}{2} \frac{\partial^{2} \psi_{x}(x, z)}{\partial x^{2}}+U_{x}(x, z) \psi_{x}(x, z)
$$

where $z=c t$ is the longitudinal distance of the beam movement and $U_{x}(x, z) \equiv u_{x}(x, z) / m_{0} \gamma_{r} \beta_{r}^{2} c^{2}$ is the dimensionless potential with the relativistic parameters, $\beta_{r}=v / c$ and $\gamma_{r}=(1-$ $\left.\beta_{r}^{2}\right)^{-1 / 2}$. The parameter $\epsilon_{x}$ is related to the emittance of the particle distribution in the $x-x^{\prime}$ space, which is explained later. The transverse particle distribution can be obtained by the squared magnitude of the beam wave function, $N\left|\psi_{x}(x, z)\right|^{2}$ with the particle number of $N$. In this convention, the beam wave function satisfies the normalization condition as follows, $\int_{-\infty}^{\infty}\left|\psi_{x}(x, z)\right|^{2} d x=1$. The corresponding equation for the time evolution of the wave function in the $y$ direction can be obtained by replacing $x$ with $y$ in Eq. (11). We note that the time evolution of the beam wave function in $y$ direction is independent of that in the $x$ direction because the one dimensional Schrödinger-type equation in the $y$ direction includes the different parameter of $\epsilon_{y}$ and we considered the linear external forces only. In the following analysis, the parameters and the functions in the $x$ direction are used without subscript if there is no confusion.

We can solve the differential equation by imposing the following two boundary conditions, $\sigma^{2}(z=0)=\sigma_{0}^{2}$ and $\left.\frac{1}{\sigma} \frac{d \sigma}{d z}\right|_{z=0}=\frac{1}{\rho_{0}}[5]$. The $\sigma$ denotes the root mean square (rms) size 
of the beam distribution and $\rho$ is the curvature radius of the beam wave function along the beam direction.

Another efficient way to solve the differential equation is known as the functional integral method [7] where the resulting wave function is given by the product of a kernel (or propagator) and the initial beam wave function,

$$
\psi\left(x_{f}, z_{f}\right)=\int_{-\infty}^{\infty} d x_{i} K\left(x_{f}, z_{f} ; x_{i}, z_{i}\right) \psi\left(x_{i}, z_{i}\right)
$$

Since a kernel represents an optical element like a quadrupole magnet or a drift space, the functional method can separate a multi-components problem into several single-component problems. This property is the main advantage of this functional method in the thermal wave model.

We can obtain the kernels from the path integral method [7] directly as follows,

$$
K\left(x_{f}, z_{f} ; x_{i}, z_{i}\right)=\int \mathcal{D}[x(z)] e^{i S(z) / \epsilon},
$$

where $S(z)=\int_{z_{i}}^{z_{f}} d z \mathcal{L}\left(x(z), x^{\prime}(z)\right)$ is called the action. The Lagrangian, $\mathcal{L}$, of a system is the difference of the kinetic and potential energy terms.

In this work, we will restrict our attention to a system consisting of quadrupole magnets and drift spaces. The potential energy terms of the beam optical elements are given by

$$
U(x)= \begin{cases}0 & \text { for a drift space, } \\ \frac{1}{2} k_{1} x^{2} & \text { for a focusing quadrupole magnet }\end{cases}
$$

where $k_{1}$ is positive in the focusing case. The potential term for the defocusing magnet is $-\left(k_{1} / 2\right) x^{2}$.

The kernel, $K_{0}$, for a drift space which has no potential term is given by

$$
K_{0}\left(x_{f}, z_{f} ; x_{i}, z_{i}\right)=\left(\frac{1}{2 \pi i \epsilon\left(z_{f}-z_{i}\right)}\right)^{1 / 2} e^{\frac{i}{2 \epsilon\left(z_{f}-z_{i}\right)}\left(x_{f}-x_{i}\right)^{2}} .
$$

The kernel, $K_{f}$, for the focusing quadrupole magnet is given by

$$
K_{f}\left(x_{f}, z_{f} ; x_{i}, z_{i}\right)=\left(\frac{\sqrt{k_{1}}}{2 \pi i \epsilon \sin \left(\sqrt{k_{1}}\left(z_{f}-z_{i}\right)\right)}\right)^{1 / 2} e^{i \frac{\sqrt{k_{1}}}{2 \epsilon}\left[\left(x_{f}^{2}+x_{i}^{2}\right) \cot \sqrt{k_{1}} z-2 x_{f} x_{i} \csc \sqrt{k_{1}} z\right]} .
$$

For the defocusing case, the kernel is obtained easily by replacing the cot and csc functions in Eq.(6) with coth and csch functions, respectively.

Since the potential energy terms are related to linear forces only, the integration in Eq. (2) becomes very simple if the initial beam wave function is a Gaussian-type such as

$$
\psi_{1}(x, 0)=\left(\frac{1}{2 \pi \sigma_{1}^{2}}\right)^{\frac{1}{4}} \exp \left[-\frac{x^{2}}{4 \sigma_{1}^{2}}+i\left(\frac{x^{2}}{2 \epsilon \rho_{1}}+\theta_{1}\right)\right]
$$

where $\sigma_{1}, \rho_{1}, \theta_{1}$ are the initial values of the rms beam size, the curvature radius, and the input phase, respectively.

After the input beam passes through a linear optical element, the beam wave function remains the Gaussian-type such as

$$
\psi_{2}(x, z)=\left(\frac{1}{2 \pi \sigma_{2}^{2}(z)}\right)^{\frac{1}{4}} \exp \left[-\frac{x^{2}}{4 \sigma_{2}^{2}(z)}+i\left(\frac{x^{2}}{2 \epsilon \rho_{2}(z)}+\theta_{1}+\theta_{2}(z)\right)\right] .
$$


The different forms of the parameter functions, $\theta_{2}(z), \sigma_{2}(z)$, and $\rho_{2}(z)$, characterize the properties of each optical element.

In a drift space, the functions are given by

$$
\begin{aligned}
\sigma_{2}^{2}(z) & =\sigma_{1}^{2}\left[\left(\frac{\epsilon z}{2 \sigma_{1}^{2}}\right)^{2}+\left(1+\frac{z}{\rho_{1}}\right)^{2}\right], \\
\tan 2 \theta_{2}(z) & =-\frac{\epsilon}{2 \sigma_{1}^{2}} \frac{z \rho_{1}}{z+\rho_{1}}, \\
\frac{1}{\rho_{2}(z)} & =\frac{1}{\rho_{1}}\left[\frac{\rho_{1}}{z}-\left(\frac{\sigma_{1}}{\sigma_{2}(z)}\right)^{2}\left(1+\frac{\rho_{1}}{z}\right)\right] .
\end{aligned}
$$

In a focusing quadrupole magnet, they are given by

$$
\begin{aligned}
\sigma_{2}^{2}(z) & =\sigma_{1}^{2}\left[\left(\cos \left(\sqrt{k_{1}} z\right)+\frac{1}{\sqrt{k_{1}} \rho_{1}} \sin \left(\sqrt{k_{1}} z\right)\right)^{2}+\left(\frac{\sigma_{0}}{\sigma_{1}}\right)^{4} \sin ^{2}\left(\sqrt{k_{1}} z\right)\right], \\
\tan 2 \theta_{2}(z) & =-\frac{\left(\frac{\sigma_{0}}{\sigma_{1}}\right)^{2} \sin \left(\sqrt{k_{1}} z\right)}{\cos \left(\sqrt{k_{1}} z\right)+\frac{1}{\sqrt{k_{1}} \rho_{1}} \sin \left(\sqrt{k_{1}} z\right)}, \\
\frac{1}{\rho_{2}(z)} & =\frac{1}{\rho_{1}}\left(\frac{\sigma_{1}}{\sigma_{2}(z)}\right)^{2}\left[\cos \left(2 \sqrt{k_{1}} z\right)+\frac{1}{2}\left\{\frac{1}{\sqrt{k_{1}} \rho_{1}}+\sqrt{k_{1}} \rho_{1}\left(\left(\frac{\sigma_{0}}{\sigma_{1}}\right)^{4}-1\right)\right\} \sin \left(2 \sqrt{k_{1}} z\right)\right],
\end{aligned}
$$

with $\sigma_{0}^{2}=\epsilon /\left(2 \sqrt{k_{1}}\right)$. For a defocusing lens, the functions can be obtained by replacing $\sqrt{k_{1}}$ for the focusing case with $i \sqrt{k_{1}}$. We can easily check to see if Eq. (8) is the solution of Eq. (1) by inserting the obtained beam wave function into the differential equation.

First of all, we studied how to relate the model parameters, $\sigma_{1}, \rho_{1}, \epsilon$, of the input Gaussian wave function in Eq. (77) to the twiss parameters and the unnormalized rms emittance, $\alpha_{1}, \beta_{1}, \epsilon_{r m s}$. Because $\beta$ is defined as $\sigma^{2} / \epsilon_{r m s}$ for the Gaussian distribution, we obtained $\sigma_{1}=\sqrt{\epsilon_{r m s} \beta_{1}}$. From the definition of $1 / \rho \equiv(1 / \sigma)(d \sigma / d z)$ [5], we can easily obtain $1 / \rho_{1}=-\alpha_{1} / \beta_{1}$ where we used $d \beta / d z=-2 \alpha$. Motivated by the quantum mechanical relation between the wave functions in the configuration and momentum spaces, we defined the wave function in the $x^{\prime}$ space as the Fourier transformation of the Gaussian beam wave function as follows,

$$
\begin{aligned}
\phi_{1}\left(x^{\prime}\right) & \equiv \frac{1}{\sqrt{2 \pi \epsilon}} \int_{-\infty}^{\infty} d x \exp \left[-i \frac{x x^{\prime}}{\epsilon}\right] \psi_{1}(x, 0) \\
& =\left(\frac{1}{2 \pi \sigma_{1 d}}\right) \exp \left[-\frac{\left(x^{\prime}\right)^{2}}{4 \sigma_{1 d}^{2}}+i\left\{-\frac{\left(x^{\prime}\right)^{2}}{2 \epsilon \rho_{1 d}}+\theta_{1}+\theta_{1 d}\right\}\right]
\end{aligned}
$$

where

$$
\begin{aligned}
& \sigma_{1 d}^{2}=\sigma_{1}^{2}\left[\left(\frac{\epsilon}{2 \sigma_{1}^{2}}\right)^{2}+\frac{1}{\rho_{1}^{2}}\right], \\
& \rho_{1 d}=\rho_{1}\left[\left(\frac{\epsilon}{2 \sigma_{1}^{2}}\right)^{2}+\frac{1}{\rho_{1}^{2}}\right],
\end{aligned}
$$

with $\tan \left(2 \theta_{1 d}\right)=2 \sigma_{1}^{2} /\left(\epsilon \rho_{1}\right)$. The initial particle distribution in the $x^{\prime}$ space is proportional to $\left|\phi_{1}\left(x^{\prime}\right)\right|^{2}$. Because we can define $\gamma$ as $\sigma_{d}^{2} / \epsilon_{r m s}$ for a Gaussian distribution in $x^{\prime}$ space, 
we obtain $\sigma_{1}^{2} \sigma_{1 d}^{2}=\epsilon_{r m s}^{2} \beta_{1} \gamma_{1}$. Comparing it with $\sigma_{1}^{2} \sigma_{1 d}^{2}=\epsilon^{2} / 4+\epsilon_{r m s} \alpha_{1}^{2}$ which can be obtained from Eq. (16), we can obtain $\epsilon=2 \epsilon_{r m s}$ where we used the relation between twiss parameters, $\beta_{1} \gamma_{1}-\alpha_{1}^{2}=1$. We also obtained $1 / \rho_{1 d}=-\alpha_{1} / \gamma_{1}$ from Eq. (17). We note that the relations between the model and physical parameters are valid for the wave functions at each of the beam optical elements.

We note that the above analysis for the time evolution of the beam in $x-x^{\prime}$ space is also valid in the $y-y^{\prime}$ space if we use the one-dimensional Schrödinger-type equation in $y$ direction with the emittance parameter of $\epsilon_{y}$. In the following analysis, we studied the time evolution of the beam wave functions in both the horizontal $\left(x-x^{\prime}\right)$ and vertical $\left(y-y^{\prime}\right)$ spaces by using the equations with different emittance parameters, $\epsilon_{x}$ and $\epsilon_{y}$.

In order to check on the validity of the solutions, we compared them with the PARMILA simulation results with 50,000 macro particles through the FODO lattice in the horizontal direction. It corresponds to the DOFO lattice in the vertical direction. The field gradient and effective length of the quadrupole magnets in the lattice are $10.0 \mathrm{~T} / \mathrm{m}$ and $0.2 \mathrm{~m}$, respectively. The length of the drift spaces is $0.5 \mathrm{~m}$. The particle type is proton with a kinetic energy of $100 \mathrm{MeV}$. We selected a random distribution of the particles in the trace spaces and neglected the space charge effects. The weighting function of the distribution is a Gaussian-type truncated at four times the standard deviation. Figure 1(a) (2(a) and Figure 1(b) (2(b) show the particle distributions of the input beam in the $x(y)$ and $x^{\prime}\left(y^{\prime}\right)$ directions of the horizontal (vertical) trace space, respectively. The histograms are the PARMILA result with 50,000 macro particles. The real lines represent the Gaussian input beam for the model calculation. They are obtained by fitting the histograms of the PARMILA results. In the all figures of this work, we used the same normalization factors of the distribution functions as those of the input functions. We found that the beam wave function of Eq. (15) describes the initial particle distribution very well in both $x^{\prime}$ and $y^{\prime}$ directions.

The properties of the input beam are summarized in Table1. From the relations between the model and physical parameters, we can obtain the input values of the model parameters as follows,

$$
\begin{array}{lll}
\sigma_{1 x}=0.76 \mathrm{~mm} & \rho_{1 x}=-1.48 \mathrm{~m} \quad \text { for the horizontal direction, } \\
\sigma_{1 y}=0.55 \mathrm{~mm} & \rho_{1 y}=-0.40 \mathrm{~m} & \text { for the vertical direction, } \\
k_{1}=6.74 \mathrm{~m}^{-2} & &
\end{array}
$$

where $k_{1}=q G /\left(m \gamma_{r} \beta_{r} c\right)$ with the quadrupole field gradient, $G$.

Figure 3 and Figure 4 show the particle distributions at the end of each optical element. The histograms and real lines represent the PARMILA simulation results and the model calculations in the $x$ and $x^{\prime}$ directions, respectively. Since the beam wave functions at each stage are Gaussian-type in the $x$ direction, the wave functions in the $x^{\prime}$ direction can be obtained by applying Eq. (15). Corresponding figures in the $y-y^{\prime}$ space are given in Figure 5 and Figure 6. We note that the distribution functions are proportional to the $\left|\psi_{1 x}(x)\right|^{2}\left(\left|\psi_{1 y}(y)\right|^{2}\right)$ and $\left|\phi_{1 x}\left(x^{\prime}\right)\right|^{2}\left(\left|\phi_{1 y}\left(y^{\prime}\right)\right|^{2}\right)$ in $x(y)$ and $x^{\prime}\left(y^{\prime}\right)$ directions, respectively. From Figure 4 and Figure 6, we can conclude that the Fourier transformation is a valid method to define the wave functions in the divergence directions. The figures show that the one dimensional Schrödinger-type equation of thermal wave model describes the PARMILA simulation result through the FODO (or DOFO) lattice successfully in the two dimensional trace $\left(x-x^{\prime}\right.$ or $\left.y-y^{\prime}\right)$ space. In order to check on the result quantitatively, we compared the rms beam sizes obtained by the model with the values obtained by the best-fit of the PARMILA result. It is summarized in Table 2, It shows that the model results are the same as the simulation to within $0.8 \%$. 
In conclusion, we studied the one-dimensional Schrödinger-type equation in the thermal wave model which describes the beam behavior in the $x-x^{\prime}$ or $y-y^{\prime}$ spaces. Some relations were obtained between the model parameters and physical parameters such as the twiss parameters and unnormalized rms emittance. We used a functional method in order to solve the differential equation with the Gaussian input distribution under the condition of the negligible space charge effects. The main advantage of this functional method is that we can calculate the effects of each beam optical element separately. The information of each element is summarized in a kernel. The final beam wave function of one optical element is obtained easily by the Gaussian integration of the product between the kernel and the initial Gaussian wave function. We found that there is a good agreement between the PARMILA simulation and the model calculation if we neglect the space charge effects. Even though there are some limits to the application of this method, this functional method is a very efficient tool to study the transverse beam dynamics in the thermal wave model.

\section{ACKNOWLEDGEMENTS}

This work is supported by the $21 \mathrm{C}$ Frontier R\&D program in the Ministry of Science and Technology of the Korean government.

\section{References}

[1] R. Fedele and G. Miele, Nuovo Cimento D 13 (1991) 1527.

[2] R. Fedele and P. K. Shukla, Phys. Rev. A 45 (1992) 4045.

[3] R. Fedele and G. Miele, Phys. Rev. A 46 (1992) 6634.

[4] S. A. Khan and M. Pusterla, Eur. Phys. J. A 7 (2000) 583.

[5] R. Fedele, F. Galluccio and G. Miele, Phys. Lett. A 185 (1994) 93.

[6] R. Fedele, F. Galluccio, V. I. Man'ko and G. Miele, Phys. Lett. A 209 (1995) 263.

[7] H. Holstein, Topics in Advanced Quantum Mechanics (Addison-Wesley, 1992).

[8] H. Takeda and J. Billen, PARMILA, LA-UR-98-4478. 
Table 1: The twiss parameters and unnormalized rms emittances of the input beam in the horizontal and vertical directions.

\begin{tabular}{l|ccc}
\hline & $\alpha$ & $\beta(\mathrm{m} / \mathrm{rad})$ & $\epsilon_{r m s}\left(10^{-7} \mathrm{~m}-\mathrm{rad}\right)$ \\
\hline horizontal $(x)$ axis & 1.62 & 2.41 & 2.46 \\
vertical $(y)$ axis & 2.87 & 1.16 & 2.57 \\
\hline
\end{tabular}

Table 2: The rms beam sizes obtained by the model and the best fit of the PARMILA result in the horizontal and vertical (numbers in parentheses) directions.

\begin{tabular}{l|ccc}
\hline & model $(\mathrm{mm})$ & PARMILA $(\mathrm{mm})$ & deviation (\%) \\
\hline After a F(D) lattice & $0.572(0.353)$ & $0.575(0.355)$ & $-0.52(-0.56)$ \\
After a drift space & $0.251(0.369)$ & $0.253(0.370)$ & $-0.79(-0.27)$ \\
After a D(F) lattice & $0.554(0.460)$ & $0.558(0.461)$ & $-0.72(-0.22)$ \\
After a drift space & $1.521(0.644)$ & $1.530(0.647)$ & $-0.59(-0.46)$ \\
\hline
\end{tabular}




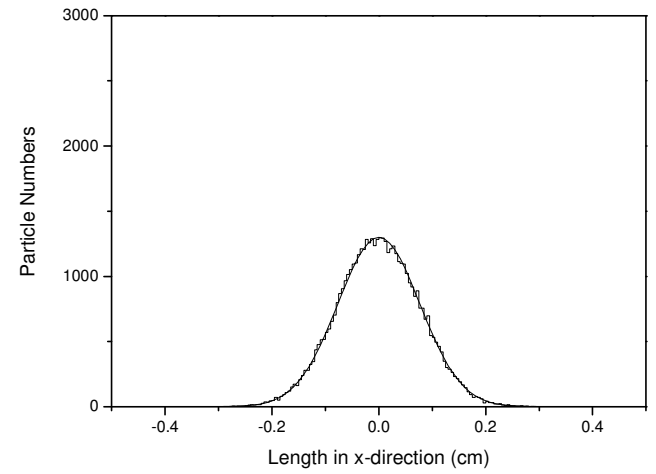

(a)

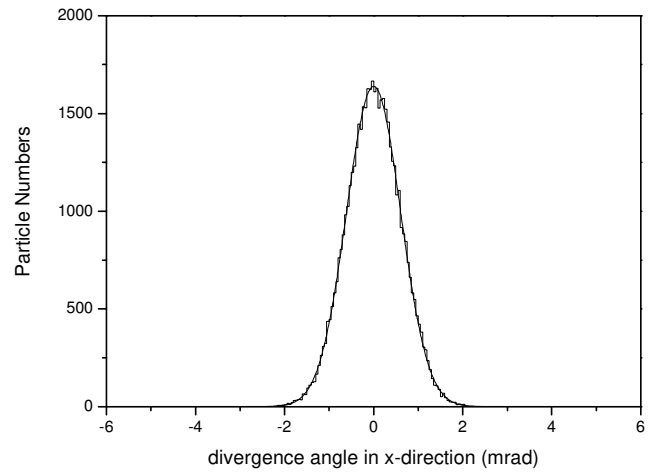

(b)

Figure 1: Particle distributions of the input beam in (a) $x$-direction and (b) $x^{\prime}$-direction of the horizontal trace space. The histograms and real lines represent the PARMILA results and the model predictions, respectively.

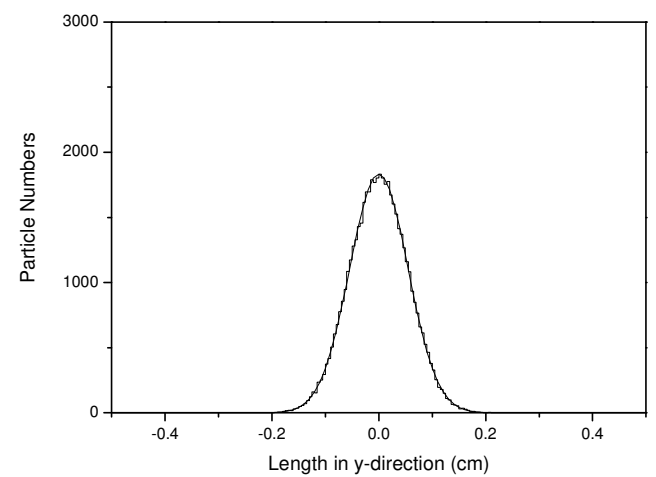

(a)

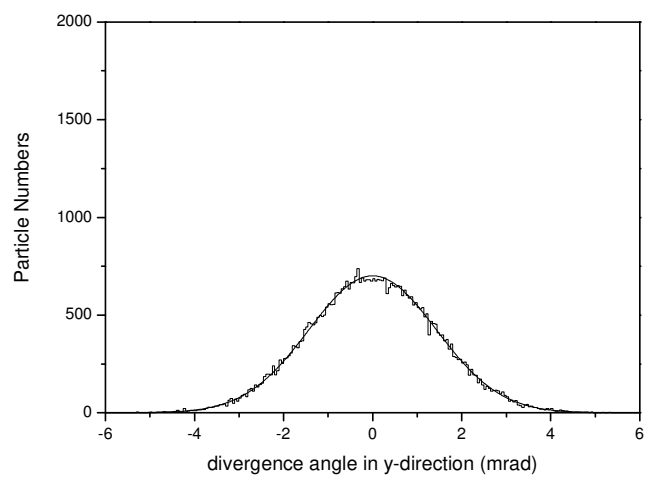

(b)

Figure 2: Particle distributions of the input beam in (a) $y$-direction and (b) $y^{\prime}$-direction of the vertical trace space. The histograms and real lines represent the PARMILA results and the model predictions, respectively. 


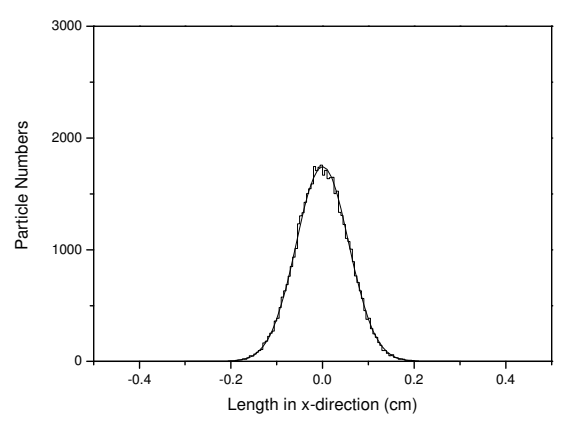

(a)

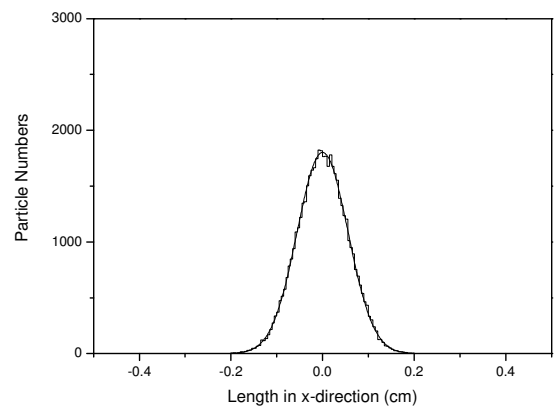

(c)

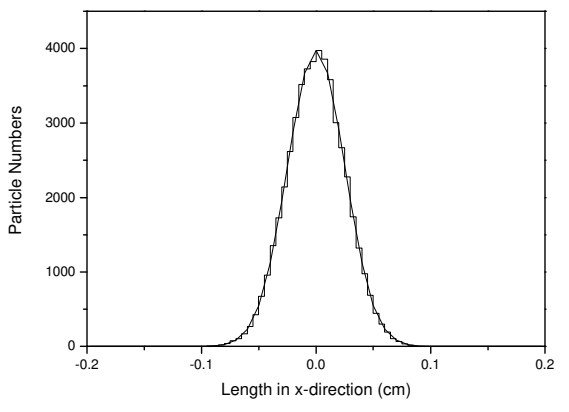

(b)

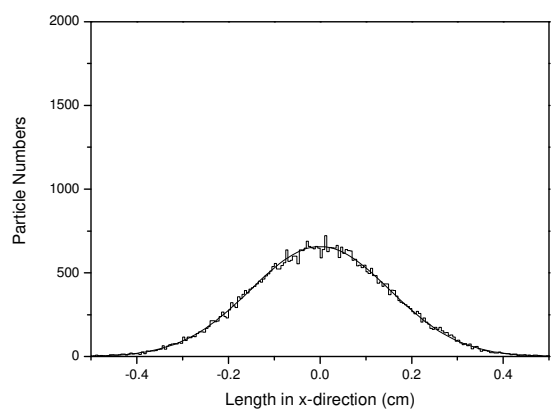

(d)

Figure 3: Particle distributions on the horizontal axis with histograms for the PARMILA results and real lines for the model calculations: (a) after a focusing quadrupole (b) after a drift space (c) after a defocusing quadrupole (d) after a drift space using the same normalization as the input beam distribution function. 


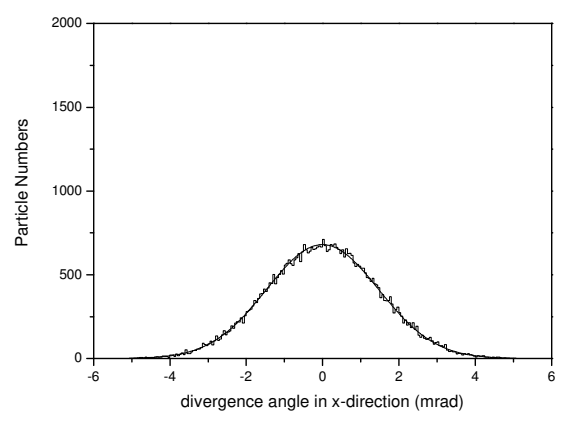

(a)

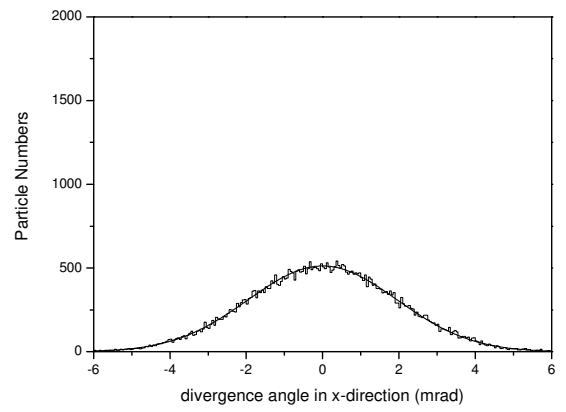

(c)

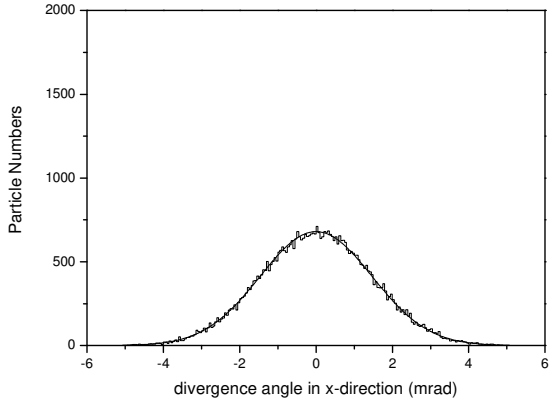

(b)

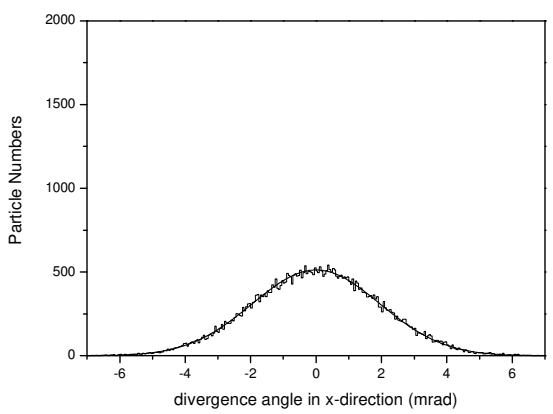

(d)

Figure 4: Particle distributions on the horizontal divergence axis with histograms for the PARMILA results and real lines for the model calculations using Fourier transformation of the beam wave function : (a) after a focusing quadrupole (b) after a drift space (c) after a defocusing quadrupole (d) after a drift space using the same normalization as the input beam distribution function. 


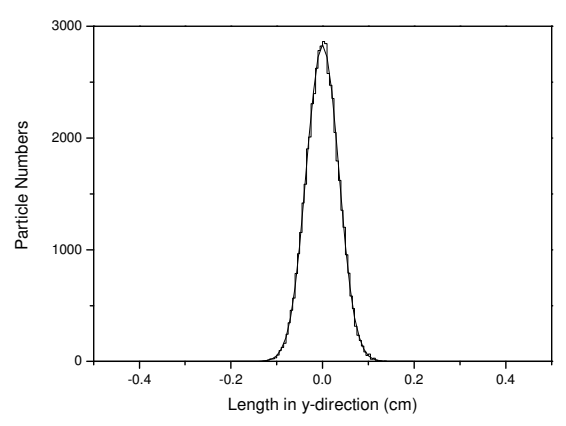

(a)

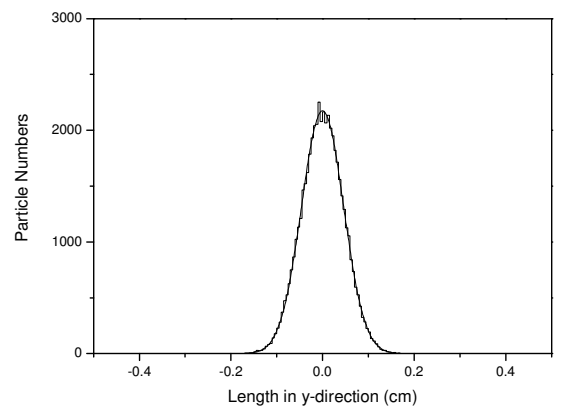

(c)

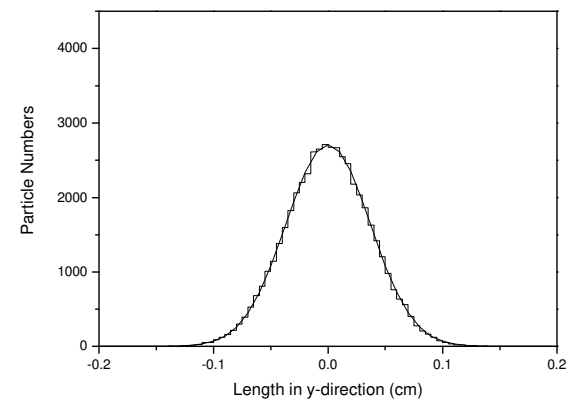

(b)

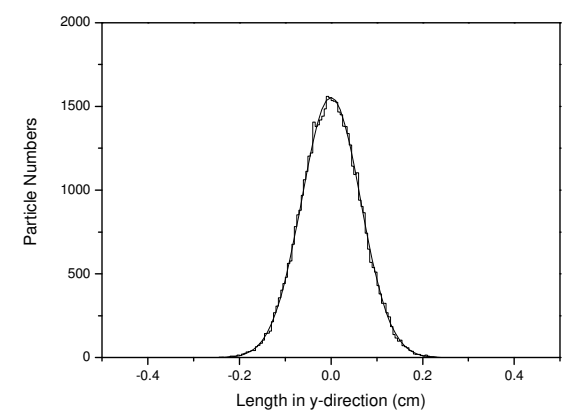

(d)

Figure 5: Particle distributions on the vertical axis with histograms for the PARMILA results and real lines for the model calculations: (a) after a defocusing quadrupole (b) after a drift space (c) after a focusing quadrupole (d) after a drift space using the same normalization as the input beam distribution function. 


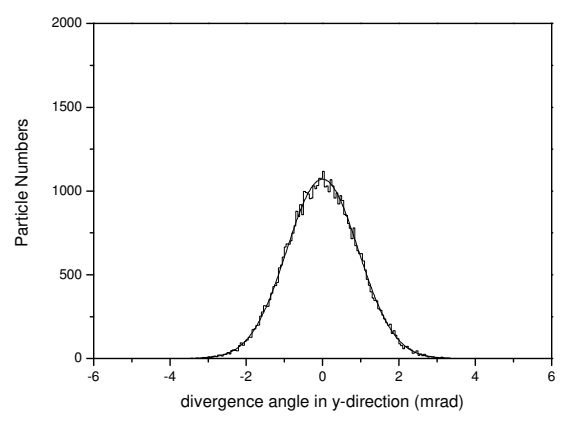

(a)

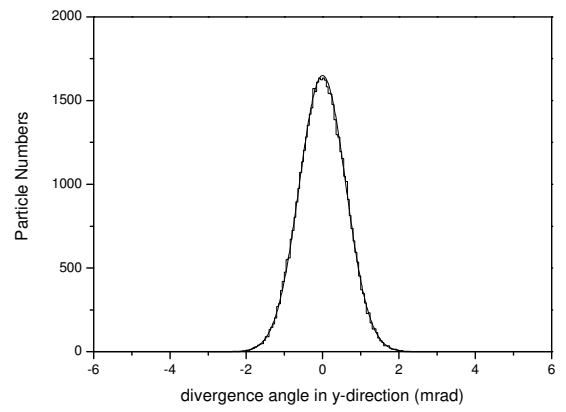

(c)

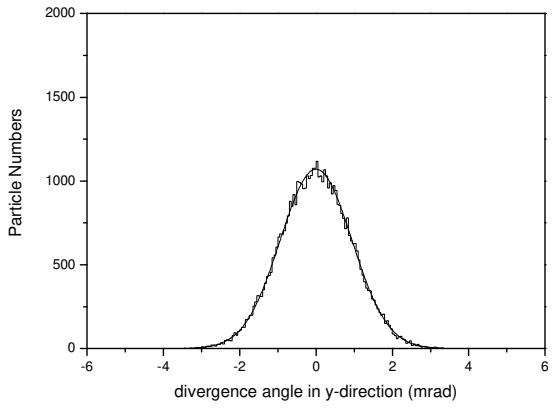

(b)

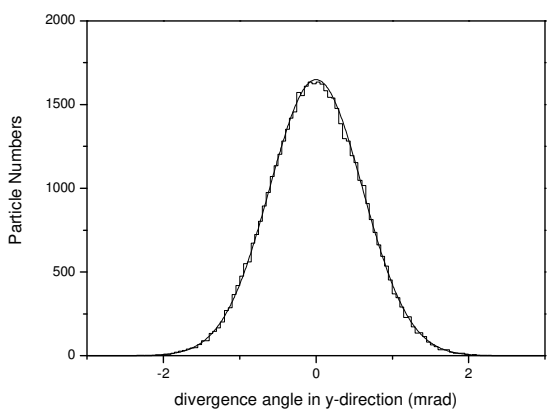

(d)

Figure 6: Particle distributions on the vertical divergence axis with histograms for the PARMILA results and real lines for the model calculations using Fourier transformation of the beam wave function : (a) after a defocusing quadrupole (b) after a drift space (c) after a focusing quadrupole (d) after a drift space using the same normalization as the input beam distribution function. 